\title{
High Mycobacterium bovis genetic diversity in a low prevalence setting
}

\author{
A.L. Michel ${ }^{\text {a,* }}$, T.M. Hlokwe ${ }^{\text {a }}$, M.L. Coetzee ${ }^{\text {a,1 }}$, L. Maré ${ }^{\text {a,2 }}$, \\ L. Connoway ${ }^{b}$, V.P.M.G. Rutten ${ }^{\text {c }}$, K. Kremer ${ }^{\mathrm{d}}$ \\ ${ }^{a}$ Tuberculosis Laboratory of the ARC-Onderstepoort Veterinary Institute, Private Bag x05, Onderstepoort 0110, South Africa \\ ${ }^{\mathrm{b}}$ Directorate Veterinary Services, Mpumalanga Province, South Africa \\ ${ }^{\mathrm{c}}$ Department of Infectious Diseases and Immunology, Utrecht University, Yalelaan 1, 3584 CL Utrecht, The Netherlands \\ ${ }^{\mathrm{d}}$ National Mycobacteria Reference Laboratory, National Institute of Public Health and the Environment, \\ Bilthoven, P.O. Box 1, 3720 BA Bilthoven, The Netherlands
}

Received 6 March 2007; received in revised form 16 July 2007; accepted 18 July 2007

\begin{abstract}
The genetic diversity among South African Mycobacterium bovis isolates from cattle was determined by genetic fingerprinting. The restriction fragment length polymorphism (RFLP) markers IS6110 and polymorphic GC-rich sequence (PGRS) as well as spoligotyping and determination of variable number of tandem repeats (VNTR) were used to characterize sub samples of $91 \mathrm{M}$. bovis field isolates. PGRS RFLP was the single most discriminatory method and combinations of typing methods, which included IS6110 and/or PGRS had the highest discriminatory power, able to reveal 29 distinct genotypes among 35 farms with no epidemiological link. Three of the farms were co-infected with two genetically unrelated strains.

In contrast to reports from European and also other colonised countries on the African continent our findings are suggestive of a high genetic diversity of M. bovis in South Africa's cattle population, implying a variety of unrelated ancestor strains. Despite effective intervention through test-and-slaughter campaigns no indication of a 'founder effect' was apparent in the panel of isolates derived from all infected provinces.
\end{abstract}

(C) 2007 Elsevier B.V. All rights reserved.

Keywords: Mycobacterium bovis; Cattle; RFLP; IS6110; PGRS; Spoligotyping; VNTR typing; Genetic diversity

* Corresponding author. Tel.: +27 125299384;

fax: +27 125299127 .

E-mail address: MichelA@arc.agric.za (A.L. Michel).

${ }^{1}$ Current address: Deltamune (PTY) Ltd., Centurion, South Africa.

${ }^{2}$ Current address: ARC-Animal Production Institute, Irene, South Africa.

\section{Introduction}

Bovine tuberculosis is believed to have been introduced into South Africa and possibly the sub region by European settlers (Hutcheon, 1880). In addition, cattle imports from Australia, Argentina and Madagascar in the early 20th century were reported to often include infected animals (Cousins 
et al., 2004). The introduction of a National Tuberculosis Scheme in 1969 resulted in the reduction of infected commercial cattle herds from $11.85 \%$ in 1971 to $0.39 \%$ in 1995 , but ever since sporadic outbreaks continued to occur. Between 1993 and 2005 a total of 209 outbreaks were reported, with a peak of 20 outbreaks in 2003 (Animal disease statistics, National Department of Agriculture). Presently, the control of bovine tuberculosis is based on intradermal tuberculin testing and slaughter as well as on abattoir surveillance. Genetic typing of Mycobacterium bovis has contributed to a greatly improved knowledge of inter-bovine and interspecies transmission of bovine tuberculosis (Durr et al., 2000). This understanding is crucial to the effective management of bovine tuberculosis control schemes and the wildlife-livestock interface in countries where wildlife reservoirs for $M$. bovis have been identified, including South Africa (Haddad et al., 2004; Skuce and Neill, 2001). In the absence of a 'best' technique, the most widely used DNA typing techniques for $M$. bovis include IS6110 and PGRS restriction fragment length polymorphism (RFLP) typing, spoligotyping and variable number of tandem repeat (VNTR) typing (Van Soolingen, 2001). In this study these techniques were used to conduct the first comparative genetic analysis of $M$. bovis isolates from South African cattle. We aimed at determining the genetic diversity of $M$. bovis among domestic cattle in South Africa using established typing methods with proven reproducibility in our setting.

\section{Materials and methods}

\subsection{Sample collection}

A total of $90 \mathrm{M}$. bovis isolates from 42 South African cattle herds in six provinces and one additional isolate from Swaziland were used in this study. Thirty-nine isolates were derived from a field investigation in the year 2003 into a multiple farm outbreak assumed to involve 12 farms (Mpekwane et al., 2004). Fifty-two M. bovis isolates from 35 infected farms in all six provinces with known occurrence of bovine tuberculosis had been collected as a result of routine sample submissions by state veterinary officials between 1993 and 2000. This represents $23 \%$ of the 148 outbreaks reported in South Africa during this period. No selection took place but all viable $M$. bovis isolates available in the laboratory were used for a retrospective characterization study involving combinations of RFLP typing, spoligotyping and VNTR typing. It was postulated that the typing method or combination of methods distinguishing the highest number of known unrelated outbreak strains would be considered the most discriminative approach. To facilitate this interpretation within the epidemiological context, isolates were classified into the following panels and evaluated against their specific background:

\subsubsection{Isolates from epidemiologically related herds}

2.1.1.1. Isolates from the same herd. Between two and four isolates were analysed from each of 15 farms (farms 3, 4, 7-11, 19, 22, 25, 26, and 36-39). In addition, 28 isolates from farm 40 were examined.

\subsubsection{Isolates from different but epidemiologically} linked herds. Several months after a dispersal sale of an infected cattle herd in the Mpumalanga Province a back tracing investigation by the veterinary field officials indicated the possible spread of M. bovis to 12 different farms in various districts, and another province. Following slaughter of tuberculin reactors M. bovis was isolated from 39 cattle from eight of the suspected farms (farms 36-43) (Fig. 1).

An epidemiological link had also been suspected between two other farms. Bovine tuberculosis had been diagnosed on farm 26 which had introduced cattle from farm 27 several months before. Subsequent skin testing confirmed the presence of bovine tuberculosis infection on farm 27.

\subsubsection{Isolates from epidemiologically unrelated herds}

Thirty-three of the 35 farms sampled between 1993 and 2000 were, to the best of our knowledge, epidemiologically unrelated. Farms 26 and 27 were linked to each other but not any other farm. The eight herds described under Section 2.1.1.2 were considered as one epidemiological unit and unrelated to the other 34 unlinked herds. The total number of outbreaks considered unrelated was 35 . 


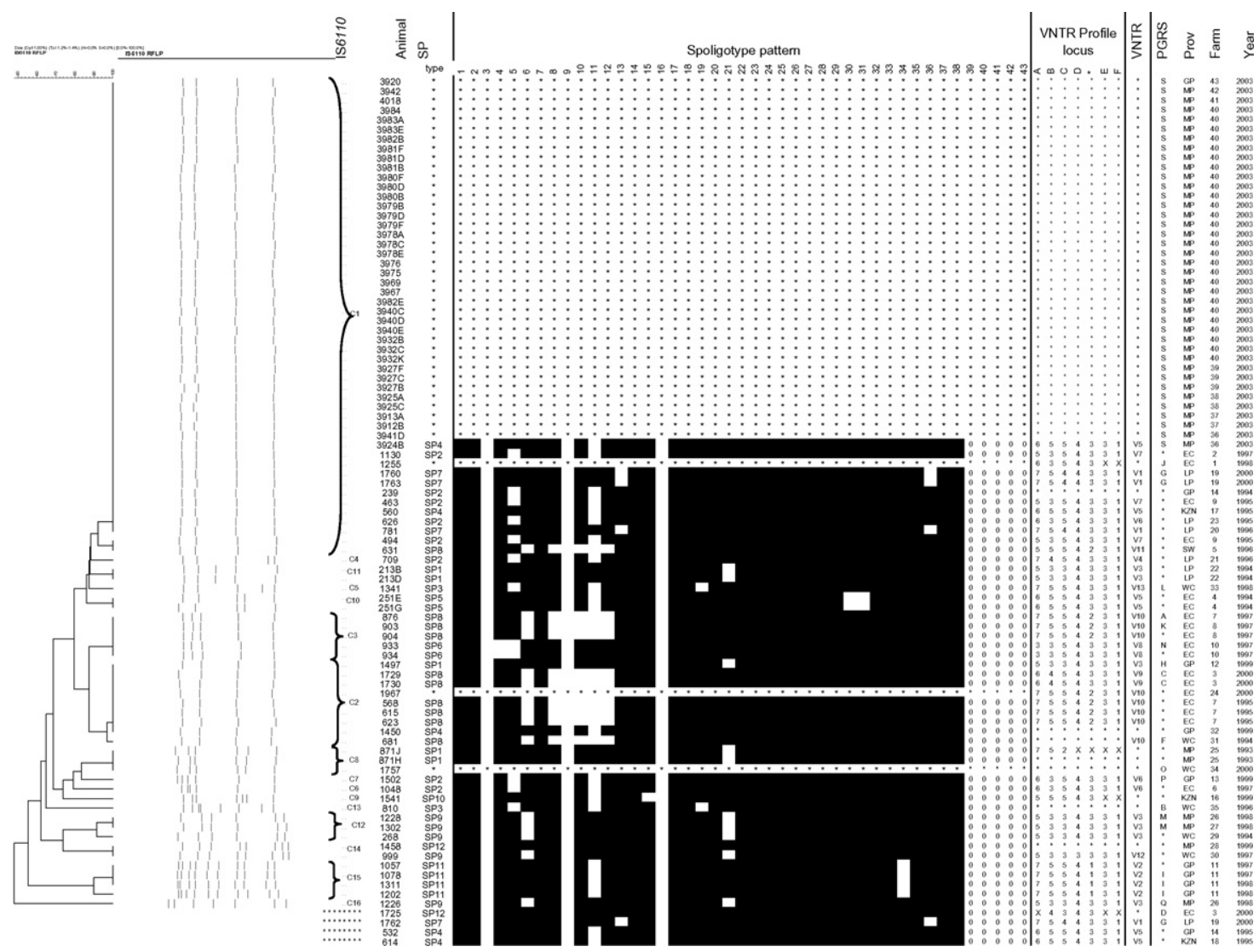

Fig. 1. DNA fingerprint results of $91 \mathrm{M}$. bovis isolates from cattle ordered by IS6110 RFLP similarity, followed by spoligopatterns, VNTR profiles and PGRS RFLP types as well as farm identifications, province of origin, year of collection. *Data not available. MP: Mpumalanga Province; EC: Eastern Cape Province; GP: Gauteng Province; LP: Limpopo Province; KZN: Kwazulu/Natal Province; and WC: Western Cape Province.

\subsection{Bacterial isolation}

All samples were processed in the Tuberculosis Laboratory of the ARC-Onderstepoort Veterinary Institute for culture according to standard procedures (Bengis et al., 1996; Alexander et al., 2002). Pure subcultures from all M. bovis isolates on LöwensteinJensen medium containing pyruvate were routinely stored at $-20{ }^{\circ} \mathrm{C}$.

\subsection{DNA extraction}

DNA extraction from $M$. bovis isolates was accomplished from colonies of either fresh or revived subcultures on Löwenstein-Jensen medium with pyruvate. Following heat-inactivation at $80^{\circ} \mathrm{C}$ for $60 \mathrm{~min}$, the colonies were scraped off and suspended in $5 \mathrm{ml}$ of extraction buffer $(50 \mathrm{~g} / \mathrm{l}$ mono sodium glutamic acid; $6.06 \mathrm{~g} / \mathrm{l}$ Tris- $\mathrm{HCl}(\mathrm{pH} 7.4) ; 9.3 \mathrm{~g} / \mathrm{l}$ EDTA) (Warren, personal communication), to which lysozyme $(50 \mathrm{mg} / \mathrm{ml})$ and RNAseA $(10 \mathrm{mg} / \mathrm{ml})$ were added. The suspensions were incubated for $2 \mathrm{~h}$ at $37{ }^{\circ} \mathrm{C}$ after which proteinase $\mathrm{K}$ at a final concentration of $0.5 \mathrm{mg} / \mathrm{ml}$ was added. After incubation at $45^{\circ} \mathrm{C}$ overnight phenol/chloroform extraction was performed. The final pellet was resuspended in $40 \mu \mathrm{l}$ TE (1 mM Tris-HCl (pH 7.6); 0.1 mM EDTA).

\subsection{Genetic typing}

\subsubsection{IS6110 RFLP typing}

For IS6110 RFLP typing approximately $1.5 \mu \mathrm{g}$ of mycobacterial DNA was digested overnight with 1.5 units of PvuII. Subsequently, the resulting frag- 
ments were separated by electrophoresis on a $0.8 \%$ agarose gel. DIG-labelled molecular weight size marker VII (Roche) was loaded in the first, middle and last lane of the gel. Southern blot transfer was performed as described by Skuce et al. (1994). IS6110-containing DNA fragments were detected through hybridization with the entire IS6110 sequence as a probe, according to the manufacturer's instructions (Roche Molecular Biochemicals-The DIG System User's Guide, 1995).

2.4.1.1. Analysis of IS6110 RFLP patterns. The GelCompar II software (Applied Maths, Sint-Martens-Latem, Belgium) was used to determine the level of similarity between the IS6110 RFLP patterns by using the similarity coefficient of Dice and the unweighted pair group method with arithmetic averages (UPGMA) for clustering maximum tolerance 1.2\%). DIG-labelled Molecular Weight Marker VII (Roche Diagnostics) was used as external marker for normalisation. Strains with a similarity coefficient of greater than $90 \%$ were considered identical provided they contained the same number of bands.

\subsubsection{PGRS RFLP typing}

For PGRS RFLP typing $1.5 \mu \mathrm{g}$ of $M$. bovis DNA was digested with AluI and electrophoresis of DNA fragments was performed on a $1.2 \%$ agarose gel (Cousins et al., 1998). Southern blotting and detection after hybridization at $55{ }^{\circ} \mathrm{C}$ with a DIG-labelled PGRS oligonucleotide probe (5'-GTC GTC AGA CCC AAA ACC CCG AGA GGG GAC GGA AAC- $3^{\prime}$ ) were performed according to the manufacturer's instructions (Roche Diagnostics). Banding patterns were analysed manually.

\subsubsection{Spoligotyping}

Spoligotyping was performed according to the protocol of Kamerbeek et al. (1997). With this method, the direct repeat region of the isolates was amplified by PCR, and the PCR products were hybridized to 43 oligonucleotides of known spacer sequences by reversed-line blot hybridization. Because one of the primers for PCR was biotin labelled, and hence the PCR product, the presence of spacers was detected after incubation with streptavidin-peroxidase and enhanced chemiluminescence detection (ECL Detection kit, Amersham Bios- ciences). The spoligopatterns were compared and assigned a $M$. bovis spoligotype number (SP number). The spoligopatterns were also compared to the international database on http://www.mbovis.org and new patterns were reported and assigned a unique SB code.

\subsubsection{VNTR typing}

VNTR typing was performed according to the method of Frothingham and Meeker-O'Connell (1998), at the Veterinary Laboratory Agencies Weybridge, United Kingdom. Primer pairs for ETR loci A to F were similar to those published previously (Frothingham and Meeker-O'Connell, 1998), except for the following minor changes: the ETR-B forward primer had a GGTT extension and the ETR-B reverse primer had a CTATA extension to improve annealing. The ETR-F forward and reverse primers were shortened by 3 and $4 \mathrm{bp}$, respectively, at the $5^{\prime}$ side of the probes (ETR-B forward: 5'-GCGAACACCAGGACAGCATCATGGGTT-3', ETR-B reverse: 5'-GGCATGCCGGTGATCGAGTGGCTATA-3', ETR-F forward: 5'-GGTGATGGT CCGGCCGGTCAC- $3^{\prime}$ and ETR-F reverse 5'-GTGCTCGACAACGCCATGCC-3'). Each PCR reaction consisted of $10 \mu \mathrm{l}$ of Qiagen Hotstar Mastermix (Qiagen), $0.5 \mu \mathrm{l}$ of each of the primers for each locus (at $10 \mathrm{pmol} / \mu \mathrm{l}$ for all primers except for ETR-A primers which were used at $20 \mathrm{pmol} / \mu \mathrm{l}$ ) and $2 \mu \mathrm{l}$ of heat-killed cell supernatant in a final volume of $20 \mu \mathrm{l}$. Following an initial denaturation at $94{ }^{\circ} \mathrm{C}$ for $15 \mathrm{~min}$ each sample was subjected to 30 cycles of $94{ }^{\circ} \mathrm{C}$ for $30 \mathrm{~s}, 68{ }^{\circ} \mathrm{C}$ for $60 \mathrm{~s}$, and $72{ }^{\circ} \mathrm{C}$ for $2 \mathrm{~min}$. Followed by an extended annealing temperature of $72{ }^{\circ} \mathrm{C}$ for $10 \mathrm{~min}$. Heat-killed cell supernatants of $M$. tuberculosis H37RV were used in each set of reactions as a positive control. PCR products were separated on a ABI 377 Sequencer, and analysed using ABI Prism Genescan software. The sizes of VNTR loci alleles were estimated by comparison to a ROX size standard (Applied Biosystems). The PCR products were compared to size standards and converted to repeat numbers at each locus using standard allele naming tables (ABI Genotyper software).

The VNTR genotype of a strain, representing the number of repeat elements at each locus, is presented as a series of integers representing the A to F VNTR loci, respectively. The ETR-D locus contains a $24 \mathrm{bp}$ deletion in one of the repeats and the naming 
convention indicates the presence of this deletion by a * i.e. $4^{*}(=3 \times 77 \mathrm{bp}$ repeats and one $53 \mathrm{bp}$ repeat $)$. The ETR-F locus contains $79 \mathrm{bp}$ tandem repeats and $55 \mathrm{bp}$ tandem repeats. The naming convention indicates the number of $79 \mathrm{bp}$ repeats followed by $55 \mathrm{bp}$ repeats separated by a period.

\section{Results}

\subsection{Identification of bacterial isolates}

All isolates from cattle tissues were identified as $M$. bovis by either confirming the biochemical characteristics of microaerophilic growth, lack of niacin production and nitratase reductase, and pyrazinamide resistance or, alternatively, by PCR amplification of targets specific for the M. tuberculosis complex and $M$. bovis, in particular.

\subsection{Genetic typing}

IS6110 and PGRS RFLP analysis, spoligotyping and VNTR typing were applied to different subsamples of $92 \mathrm{M}$. bovis isolates. For most isolates typing procedures could not be synchronized but had to be performed partially on frozen and revived cultures. This is considered the most likely cause of failures to obtain sufficient DNA of good quality for typing. Complete analysis involving all four typing methods was carried out on 17 isolates from 12 farms, while only three or less typing methods could be applied to the remaining isolates. Results are summarized in Fig. 1.

\subsubsection{Isolates from epidemiologically related herds}

3.2.1.1. Isolates from the same herd. Genetic typing rendered identical typing patterns for multiple isolates from the same farm in 15 of the 16 herds in this panel. With the exception of two farms this was true for all "same herd" isolates. Both in herds 3 and 7 one strain was isolated which had a genotype that did not match the genotype of the isolates of the herd mates, suggesting the co-existence of two M. bovis strains in those herds. In the case of farm 3, the PGRS RFLP pattern as well as spoligotyping data of isolate 1725 differed from those of the other isolates from this farm.
In the case of farm 7, isolate 876 could be distinguished by an additional band in the IS6110 RFLP pattern, but not by spoligotyping nor VNTR typing (PGRS RFLP typing data lacked). In contrast, the only two isolates from farm 26 (1228 and 1226) were found to be unrelated regarding both the PGRS and IS6110 RFLP patterns (similarity coefficient of 0.60 ). Unique DNA fingerprints remained unchanged for the period between samplings from the same herd, which was on average 2 years (see Fig. 1).

\subsubsection{Isolates from different but epidemiologically} linked herds. The genetic relatedness of 39 isolates from eight infected herds, assumed to be linked through a dispersal sale of an infected cattle herd, was investigated. Initial IS6110 RFLP analysis grouped all samples in the genotype $\mathrm{C} 1$, which contains only two IS copies (Fig. 1, farms 36-43). In subsequent PGRS RFLP typing all isolates again displayed an identical PGRS banding pattern, hence supporting the outcome of the epidemiological field investigation of a common source of infection for all eight farms.

Because of cattle movement from farms 27-26, an epidemiological link was also suspected between these two farms. Indeed, the PGRS and IS6110 RFLP patterns of one of the isolates from farm 26 (isolate 1228) were identical to those of the isolate of farm 27 (isolate 1302), confirming the epidemiological link (Fig. 1).

\subsubsection{Genetic profiles among epidemiologically unrelated $M$. bovis isolates}

M. bovis strains isolated from herds with no known link are assumed to be genetically different. This section therefore served to evaluate the results for the various typing methods against this hypothesis. The study identified 29 genetically distinct $M$. bovis strains among 35 herds with no known epidemiological link (Table 1). The highest level of discrimination was achieved when RFLP typing with IS6110 and/or PGRS was used.

\subsection{IS6110 RFLP}

Among the $49 \mathrm{M}$. bovis isolates from 34 unrelated farms subjected to IS6110 RFLP analysis, 16 distinct banding patterns were identified. These patterns comprised four to ten bands, which, due to the use 
Table 1

Comparison of the discriminatory power of different genetic markers used individually and in combination to characterize $M$. bovis isolates from epidemiologically unrelated farms

\begin{tabular}{lll}
\hline Typing method(s) used in parallel & $\begin{array}{l}\text { Number of unrelated } \\
\text { outbreaks (isolates) analysed }\end{array}$ & $\begin{array}{l}\text { No. of genotypes } \\
\text { identified }\end{array}$ \\
\hline IS6110 + PGRS + spoligotyping + VNTR & $11(17)$ & 12 \\
IS6110 + PGRS + spoligotyping & $14(22)$ & 15 \\
PGRS + spoligotyping + VNTR & $11(18)$ & 12 \\
IS6110 + spoligotyping + VNTR & $23(39)$ & 21 \\
IS6110 + PGRS + VNTR & $11(17)$ & 12 \\
Spoligotyping + PGRS & $14(23)$ & 16 \\
Spoligotyping + VNTR & $25(41)$ & 15 \\
IS6110 + spoligotyping & $30(45)$ & 24 \\
IS6110 + PGRS & $16(24)$ & 17 \\
IS6110 + VNTR & $24(39)$ & 21 \\
IS6110 & $34(49)$ & 16 \\
VNTR & $26(42)$ & 13 \\
PGRS & $16(5)$ & 18 \\
Spoligotyping & $33(50)$ & 12 \\
Total (IS6110 and/or PGRS and/or spoligotyping and/or VNTR) & 29
\end{tabular}

${ }^{a}$ One isolate from the multiple farm outbreak (Section 2.1.1.2) was included in the analysis.

of the entire sequence of IS6110 probe, as described previously (Skuce et al., 1994), relates to two to five copies of IS6110. Unique IS6110 RFLP types, not shared with any other herd, were found for 10 infected farms (C4-C7, C9-C11, C13, C15, and C16), four IS6110 RFLP types (C3, C8, C12, C14) were shared between two or three farms, and two patterns, both resembling M. bovis strains with two copies of IS6110, were found in ten and six unrelated outbreaks, respectively (C1 and $\mathrm{C} 2$ ) (Fig. 1).

\subsection{PGRS RFLP}

PGRS RFLP typing was only applied to 25 isolates from 16 unrelated farms which yielded a total of 18 unique patterns (Fig. 1 and Table 1). All PGRS types were unique and were not found in more than one epidemiologically unlinked herd. Among the isolates of two farms (farms 3 and 26), two distinct PGRS RFLP types were observed.

\subsection{Spoligotyping}

Spoligotyping was performed on 50 isolates from 33 farms resulting in the identification of 12 spoligotypes, all of which lacked spacers 3, 9, 16, and 40-43 (Fig. 1). Seven types were each associated with several unrelated herds (SP1, SP3, SP4, SP7-SP9, and SP12), while four spoligotypes were each associated with one outbreak only (SP5, SP6, SP10, and SP11). Spoligotype SP8 was found along with SP12 on the same farm (farm 3) (see also Section 2.1).

Two of the 12 spoligopatterns identified (SP7 and SP11) had not been reported in the international $M$. bovis database before and were assigned the SB codes 1163 and 1164 (Table 2).

\subsection{VNTR typing}

VNTR typing was performed on 43 isolates resulting in 13 distinct patterns relating to 26 epidemiologically unrelated outbreaks (Table 1). As a result, seven farms revealed unique VNTR types (V2, V4, V8, V9, and V11-V13) while other types were shared by between two and five farms. Further discrimination of the more common patterns was possible if either PGRS RFLP patterns were available (V3 and V10), or if IS6110 banding patterns comprised at least six bands (V3, V5, and V6). For VNTR types V1 and V7, both detected in two herds, no further discrimination was possible due to a lack of PGRS data and corresponding low copy number IS6110 patterns. With the exception of VNTR types V3 and V5, VNTR typing revealed superior or equal differentiation between strains compared to spoligotyping. 
Table 2

$M$ bovis spoligotype and VNTR patterns frequencies among isolates in 35 unrelated cattle herds

\begin{tabular}{|c|c|c|c|c|c|c|}
\hline Spoligotype & Frequency & $\mathrm{SB} \operatorname{code}^{\mathrm{a}}$ & VNTR & Frequency & SP/VNTR & Frequency \\
\hline SP1 & 3 & SB0121 & V1 & 2 & SP4/V5 & 4 \\
\hline SP2 & 7 & SB0131 & $\mathrm{V} 2$ & 1 & SP2/V6 & 3 \\
\hline SP3 & 2 & SB0267 & V3 & 4 & SP7/V1 & 2 \\
\hline SP4 & 5 & SB0130 & V4 & 1 & SP9/V3 & 3 \\
\hline SP5 & 1 & SB0163 & V5 & 5 & SP8/V10 & 2 \\
\hline SP6 & 1 & SB0134 & V6 & 3 & $\mathrm{SP} 1 / \mathrm{V} 3$ & 2 \\
\hline SP7 & 2 & SB1163 & V7 & 2 & $\mathrm{SP} 2 / \mathrm{V} 4$ & 1 \\
\hline SP8 & 5 & SB0140 & V8 & 1 & $\mathrm{SP} 2 / \mathrm{V} 7$ & 2 \\
\hline SP9 & 3 & SB0265 & V9 & 1 & SP8/V11 & 1 \\
\hline SP10 & 1 & SB0678 & V10 & 2 & SP9/V12 & 1 \\
\hline SP11 & 1 & SB1164 & V11 & 1 & SP3/V13 & 1 \\
\hline SP12 & 2 & BCG & V12 & 1 & SP11/V2 & 1 \\
\hline $\mathrm{b}$ & $\mathrm{b}$ & b & V13 & 1 & SP5/V5 & 1 \\
\hline $\mathrm{b}$ & $\mathrm{b}$ & $\mathrm{b}$ & b & $\mathrm{b}$ & SP6/V8 & 1 \\
\hline
\end{tabular}

${ }^{\mathrm{a}}$ http://www.mbovis.org.

b End of table.

\section{Discussion}

The present study is the first to investigate DNA polymorphism among $M$. bovis isolates from cattle in South Africa. Four of the most commonly used genetic markers (IS6110 RFLP, PGRS RFLP, spoligotyping and VNTR typing) provided high levels of both, reproducibility and genetic diversity in our setting. Although the study did not permit a true comparative evaluation of the methods due to incomplete typing data for several of the isolates, we are of the opinion that the study allows first conclusions regarding the genetic diversity among South African M. bovis isolates.

We found IS6110 RFLP to be highly discriminatory for all $M$. bovis strains which contained more than three copies of the IS sequence. However, $44 \%$ of the outbreaks examined in this study were caused by a strain comprising only two copies of IS6110 (C1 or $\mathrm{C} 2$ ), hence limiting the value of this probe. PGRS RFLP was the single most discriminatory method as it was able to distinguish between all 16 epidemiologically unrelated outbreaks subjected to this method. Furthermore two of the outbreaks (farms 3 and 26) were found to be associated with two genetically different M. bovis strains, bringing the total of PGRS types identified to 18 . Previous investigators reported a similar superior performance of PGRS (Cousins et al., 1998; Van Soolingen et al., 1994). The technically much less demanding spoligotyping provided the lowest level of differentiation between strains in our study. It was possible to increase the level of discrimination of spoligotyping by second stage IS6110 RFLP, PGRS RFLP or VNTR typing, as suggested previously for $M$. tuberculosis isolates with low copy numbers of IS6110 (Rasolofo-Razanamparany et al., 2001). Both the IS6110 and PGRS RFLP typing methods confirmed transmission of infection between farms 26 and 27 as well as the co-infection of farm 26 with two genetically distinct $M$. bovis strains.

VNTR typing appeared to be less discriminatory than PGRS and IS6110 RFLP typing in our study but comparable to spoligotyping. However, certain spoligotypes and IS6110 RFLP types with three or less insertion elements could be subdivided by VNTR typing. In conclusion, the current VNTR typing protocol can provide a valuable first stage screening tool as recently suggested for $M$. tuberculosis strains (Kremer et al., 2005b). Strains with different VNTR patterns will most likely represent genetically distinct strains, but strains with the same VNTR type should be sub-typed with IS6110 RFLP typing or even PGRS RFLP typing to determine whether they represent the same clone or not. Alternatively, the resolution of VNTR typing can be increased by the number and configuration of loci most appropriate for the locally prevalent strains.

Three of the herds examined were found to be coinfected with two distinct genotypes ( 3,7 , and 26) as demonstrated by various of the typing methods 
employed. Multi-genotype infections may not be a rare event, especially in countries where bovine tuberculosis occurs at a prevalence of $>1 \%$ (Serraino et al., 1999). Costello et al. found that $10 \%$ of cattle herds examined in Ireland harboured more than one strain (Costello et al., 1999). We were unable to reliably estimate the percentage of herds with multiple strain involvement due to the small number of outbreaks analysed with all four markers. However, the fact that such events were detected in the small sample analysed in this study may either suggest a relatively high frequency of outbreaks with multiple sources of infection probably due to purchase of infected animals (Skuce et al., 1994; Neill et al., 1994) or, alternatively, persistence and evolution of "old" M. bovis strains within the country's cattle population (Milian-Suazo et al., 2002). Both scenarios appear plausible in the South African context. Despite an initial sharp decline in the bovine tuberculosis herd prevalence to below $0.4 \%$, the disease was never eradicated from the country but continued to occur and more recently the spread of the disease to all nine provinces of South Africa has been confirmed (Michel, unpublished data).

The genetic diversity detected among the $M$. bovis isolates in this study appears to be high compared to studies conducted in European countries where testand-slaughter is enforced more strictly. Genotyping of 233 M. bovis isolates from cattle in Ireland yielded 17 spoligotypes (Costello et al., 1999), Skuce et al. (2005) found 14 spoligotypes among 461 isolates of M. bovis in Northern Ireland and spoligotyping of 1349 M. bovis isolates in France identified 161 spoligotypes (Haddad et al., 2001). It has recently been reported that clonal expansion following a bovine population bottleneck is a major determinant of the reduced strain diversity of $M$. bovis in Great Britain (Smith et al., 2006). In the central African region the degree of heterogeneity appears to be low despite the absence of eradication programmes. Possible explanations are limited cattle imports from Europe and fairly recent introduction of the disease (Njanpop-Lafourcade et al., 2001; Cadmus et al., 2006; Diguimbaye-Djaibé et al., 2006). The findings of our study do not fit any of these epidemiological scenarios for South Africa. During colonial times and into the 20th century cattle imports from different European countries and other continents were respon- sible for multiple introductions of $M$. bovis and most probably a high strain heterogeneity. On the other hand, the rinderpest pandemic, killing $66 \%$ of South African cattle (Rossiter, 2004), as well as an effective 'test-andslaughter' scheme should be considered powerful population and diversity reducing factors, commonly facilitating a founder effect characterized by the establishment of a successful genotype in a geographical region (Smith et al., 2006). Our study did not present any indicators of such an effect and it may be speculated that many genotypes were only introduced in the 20th century and that incomplete eradication of outbreaks during 'test-and-slaughter' campaigns may have allowed survival of 'old' strains. In addition, it cannot be ruled out that undetected spillover of strains into wildlife occurred at the wildlife/livestock interface, possibly re-infecting cattle at a later stage.

In conclusion, the data obtained in this retrospective study show that IS6110 and PGRS RFLP represented powerful markers in revealing a high genetic diversity among cattle strains in South Africa, where the overall bovine tuberculosis prevalence is low compared to countries with a more limited strain diversity.

\section{Acknowledgements}

The authors want to thank Dr N. Smith and staff at the Veterinary Laboratories Agency Weybridge, England, for kindly performing the VNTR typing.

\section{References}

Alexander, K.A., Pleydell, E., Williams, M.C., Lane, E.P., Nyange, J.F.C., Michel, A.L., 2002. Mycobacterium tuberculosis: an emerging disease of free-ranging wildlife. Emerg. Infect. Dis. 8, 592-595.

Bengis, R.G., Kriek, N.P.J., Keet, D.F., Raath, J.P., De Vos, V., Huchzermeyer, H.F.A.K., 1996. An outbreak of bovine tuberculosis in a free-living buffalo population in the Kruger National Park, Onderstepoort. J. Vet. Res. 63, 15-18.

Cadmus, S., Palmer, S., Okker, M., Dale, J., Gover, K., Smith, N., Jahans, K., Hewinson, G., Gordon, S.V., 2006. J. Clin. Microbiol. 44, 29-34

Costello, E., O’Grady, D., Flynn, O., O’Brien, R., Rogers, M., Quigley, F., Egan, J., Griffin, J., 1999. Study of restriction fragment length polymorphism analysis and spoligotyping for epidemiological investigation of Mycobacterium bovis infection. J. Clin. Microbiol. 37, 3217-3222. 
Cousins, D., Williams, S., Liebana, E., Aranaz, A., Bunschoten, A., van Embden, J., Ellis, T., 1998. Evaluation of four DNA typing techniques in epidemiological investigations of bovine tuberculosis. J. Clin. Microbiol. 36, 168-178.

Cousins, D.V., Huchzermeyer, H.F.K.A., Griffin, J.F.T., Brueckner, G.K., van Rensburg, I.B.J., Kriek, N.P.J., 2004. Tuberculosis. In: Coetzer, J.A.W., Tustin, R.C. (Eds.), Infectious Diseases of Livestock. Oxford University Press Southern Africa, Cape Town.

Diguimbaye-Djaibé, C., Hilty, M., Ngandolo, R., Mahamat, H.H., Pfyffer, G.E., Baggi, F., Hewinson, G., Tanner, M., Zinstag, J., Schelling, E., 2006. Mycobacterium bovis isolates from tuberculous lesions in Chadian zebu cattle. Emerg. Infect. Dis. 12, 769-771.

Durr, P.A., Hewinson, R.G., Clifton-Hadley, R.S., 2000. Molecular epidemiology of bovine tuberculosis. I. Mycobacterium bovis genotyping. Rev. Sci. Technol. Off. Int. Epiz. 19, 675-688.

Frothingham, R., Meeker-O'Connell, W.A., 1998. Genetic diversity in the M. TB complex based on variable numbers of tandem DNA repeats. Microbiology 144, 1189-1196.

Haddad, N., Ostyn, A., Karoui, C., Masselot, M., Thorel, M.F., Hughes, S.L., Inwald, J., Hewinson, R.G., Durand, B., 2001. Spoligotype diversity of Mycobacterium bovis strains isolated in France from 1979 to 2000. J. Clin. Microbiol. 39, 3623-3632.

Haddad, N., Masselot, M., Durand, B., 2004. Molecular differentiation of Mycobacterium bovis isolates. Review of main techniques and applications. Res. Vet. Sci. 76, 1-18.

Hutcheon, D., 1880. Tering, consumption, tables mesenterica. Annual Report, Colonial Veterinary Surgeon, Cape of Good Hope.

Kamerbeek, J., Schouls, L., Kolk, A., van Agterveld, M., van Soolingen, D., Kuijper, S., Bunschoten, A., Molhuizen, H., Shaw, R., Goyal, M., van Embden, J., 1997. J. Clin. Microbiol. 35, 907-914.

Kremer, K., Au, B.K., Yip, P.C., Skuce, R., Supply, P., Kam, K.M., 2005b. Use of variable-number tandem-repeat typing to differentiate Mycobacterium tuberculosis Beijing family isolates from Hong Kong and comparison with IS6110 restriction fragment length polymorphism typing and spoligotyping. J. Clin. Microbiol. 43, 314-320.

Milian-Suazo, F., Banda-Ruiz, V., Ramirez-Casillas, C., ArriagaDiaz, C., 2002. Genotyping of Mycobacterium bovis by geographic location within Mexico. Prev. Vet. Med. 55, 255-264.

Mpekwane, T., Michel, A.L., Connoway, L., 2004. Preliminary results of a bovine tuberculosis outbreak investigation in $\mathrm{Mpu}-$ malanga and Gauteng Provinces using M. bovis genetic strain typing. In: Proceedings of the Annual conference of the Southern African Society of Veterinary Epidemiology and Preventive Medicine, Intundla Lodge, Pretoria, South Africa, August $25-27$.
National Department of Agriculture, Veterinary Services. Monthly and Annual disease reports www.nda.agric.za/vetweb/Animal\%20Disease/reports/. Last accessed February 21, 2006.

Neill, S.D., Pollock, J.M., Bryson, D.B., Hanna, J., 1994. Pathogenesis of Mycobacterium bovis infection in cattle. Vet. Microbiol. 40, 41-52.

Njanpop-Lafourcade, B.M., Inwald, J., Ostyn, A., Durand, B., Hughes, S., Thorel, M.F., Hewinson, G., Haddad, N., 2001. Molecular typing of Mycobacterium bovis isolates from Cameroon. J. Clin. Microbiol. 39, 222-227.

Rasolofo-Razanamparany, V., Ramarokoto, H., Aurégan, G., Gicquel, B., Chanteau, S., 2001. A combination of two genetic markers is sufficient for restriction fragment length polymorphism typing of Mycobacterium tuberculosis complex in areas with a high incidence of tuberculosis. J. Clin. Microbiol. 39, 1530-1535.

Rossiter, P.B., 2004. Rinderpest. In: Coetzer, J.A.W., Tustin, R.C. (Eds.), Infectious Diseases of Livestock. Oxford University Press Southern Africa, Cape Town.

Serraino, A., Marchetti, G., Sanguinetti, V., Rossi, M.C., Zanoni, R.G., Catozzi, L., Bandera, A., Dini, W., Mignone, W., Franzetti, F., Gori, A., 1999. Monitoring transmission of tuberculosis between wild boars and cattle: genotypical analysis of strains by molecular epidemiology techniques. J. Clin. Microbiol. 37, 2766-2771.

Skuce, R.A., Neill, S.D., 2001. Molecular epidemiology of Mycobacterium bovis: exploiting molecular data. Tuberculosis 81 , 169-175.

Skuce, R.A., Brittain, D., Hughes, M.S., Beck, L.A., Neill, S.D., 1994. Genomic fingerprinting of Mycobacterium bovis from cattle by restriction fragment length polymorphism analysis. J. Clin. Microbiol. 32, 2387-2392.

Skuce, R.A., McDowell, S.W., Mallon, T.R., Luke, B., Breadon, E.L., Lagan, P.L., McCormick, C.M., McBride, S.H., Pollock, J.M., 2005. Discrimination of isolates of Mycobacterium bovis in Northern Ireland on the basis of variable tandem repeats (VNTRs). Vet. Rec. 157, 501-504.

Smith, N.H., Gordon, S.V., de la Rua-Romenech, R., Clifton-Hadley, R.S., Hewinson, R.G., 2006. Bottlenecks and broomsticks: the molecular evolution of Mycobacterium bovis. Nat. Rev. Microbiol. 4, 670-681.

Van Soolingen, D., 2001. Molecular epidemiology of tuberculosis and other mycobacterial infections; main methodologies and achievements. Review. J. Int. Med. 249, 1-26.

Van Soolingen, D., de Haas, P.E.W., Haagsma, J., Eger, T., Hermans, P.W.M., Ritacco, V., Alito, A., van Embden, J.D.A., 1994. Use of various genetic markers in differentiation of Mycobacterium bovis strains from animals and humans and for studying epidemiology of bovine tuberculosis. J. Clin. Microbiol. 32, 2425-2433. 